\title{
Cardiovascular risk management in rheumatoid arthritis: are we still waiting for the first step?
}

\author{
Mike JL Peters ${ }^{1,2}$ and Michael T Nurmohamed ${ }^{* 1,2,3}$ \\ See related research by Desai et al., http://arthritis-research.com/content/14/6/R270
}

\begin{abstract}
Rheumatoid arthritis (RA) is associated with a similar cardiovascular risk to that in diabetes, and therefore cardiovascular risk management (CV-RM) - that is, identification and treatment of cardiovascular risk factors (CRFs) - is mandatory. However, whether and to what extent this is done in daily clinical practice is unknown. In a retrospective cohort investigation, CVRM was therefore compared between rheumatologists and primary care physicians (PCPs). Remarkably, CRFs in RA were less frequently identified and managed by rheumatologists in comparison with PCPs. In addition, PCPs assessed CRFs less frequently in RA than in diabetes. Obviously, there is a clear need for improvement of CV-RM in RA and this should be a joint effort from the rheumatologist and the PCP.
\end{abstract}

Patients with rheumatoid arthritis (RA) have an increased cardiovascular $(\mathrm{CV})$ risk that appears similar to that in diabetes. This observation highlights the significant $\mathrm{CV}$ burden in RA. In 1999, the American Diabetes Association and the American Heart Association published a statement for prevention of CV disease in diabetes. Since then, the $\mathrm{CV}$ risk in diabetes has been substantially lower than in earlier decades. Given the increased CV risk in RA, screening, identification of cardiovascular risk factors (CRFs) and cardiovascular risk management (CV-RM) are also highly needed as recommended by the European League Against Rheumatism (EULAR). The increased risk in RA is attributed to systemic inflammation as well as increased prevalence of CRFs. Hence, we should aim for tight disease control and control of CRFs.

*Correspondence: mt.nurmohamed@vumc.nl

'Department of Internal Medicine, VU University Medical Centre, PO Box 7057,

De Boelelaan 1117, 1081 HV Amsterdam, the Netherlands

Full list of author information is available at the end of the article
Presently unknown is whether and to what extent CV-RM is translated into clinical practice. In a retrospective cohort - comprising 251 patients with RA, 251 patients with diabetes, and 251 general population individuals - Desai and colleagues therefore investigated the identification and management of CRFs by rheumatologists and primary care physicians (PCPs) [1]. RA patients had to be registered at the University of Michigan Health System for at least 12 months between June 2007 and April 2012 and had been evaluated both by their rheumatologist as well as the PCP. CRFs of interest were smoking, exercise, weight, blood pressure, lipid profile, and fasting blood glucose.

In RA, PCPs identified and managed most CRFs more frequently than rheumatologists. Secondly, identification of CRFs by rheumatologists in RA patients with elevated $\mathrm{C}$-reactive protein levels was not different as compared with those with normal C-reactive protein levels. A third important observation was that PCPs identified and managed CRFs more frequently in patients with diabetes, followed by general population individuals and least often in RA patients. These striking results raise several issues.

First, it is hard to believe that the largely absent CV-RM by rheumatologists is explained by under-recognition because the increased CV risk in RA must presently be well known among rheumatologists. A large amount of literature on this topic has been published over the last decade. Additionally, the necessity to screen, identify, and manage CRFs is incorporated into training programmes for rheumatology residents [2]. Against this background, it is important to realise that there is a lag time between the publication of the EULAR guideline and its actual implementation (that is, the guideline was published in 2010 [3] while the current study started in 2007). In other words, CV-RM in today's clinical practice might have been improved, but not yet recognised.

Second, that the CV risk in RA is related to the inflammatory burden is well known. Nevertheless, the present study did not indicate that there is more attention for $\mathrm{CV}-\mathrm{RM}$ by rheumatologists in patients with a higher inflammatory load. 
Third, undertreatment of the increased $\mathrm{CV}$ risk in RA by PCPs might be explained by under-recognition because CRFs were assessed more frequently in diabetes in comparison with RA.

The EULAR guidelines recommend screening and identification of CRFs in all RA patients, and, if indicated according to $\mathrm{CV}$ risk-prediction charts, adequate management. As accurate assessment of CV risk depends on RA characteristics, the EULAR favoured individualising risk assessment. Hence, a risk multiplication factor of 1.5 should be used in the presence of two of the following criteria: disease duration $>10$ years, rheumatoid factor, and/or anti-cyclic citrullinated peptide positivity or the presence of extra-articular manifestations. However, alternative approaches have been suggested - for example, increasing the age of an RA patient by 10 years to obtain a more precise $\mathrm{CV}$ risk estimate or to use other risk scores. Perhaps this lack of an RA-specific CV riskprediction model hampers CV-RM implementation. Obviously, this discussion can only be solved by developing a RA-specific CV risk-prediction model, but this will take several years to complete.

One may obviously argue that, due to its retrospective design, the strength of the conclusions of Desai and colleagues may be limited; however, they are in line with other recently published literature and thus confirm extending evidence that CV-RM is poorly conducted in RA, both by rheumatologists and PCPs. Another argument against CV-RM in RA is that we should wait until trials have been conducted that demonstrate the efficacy of statins and antihypertensive agents in RA. However, it will be (many) years before specific risk models are available and withholding cardiopreventive drugs that are very likely to work also in our high-risk population is unethical. Moreover, it is important to realise that, due to the decreased incidence of $\mathrm{CV}$ events in the last decades, $\mathrm{CV}$ prevention trials are nowadays very difficult to conduct. For instance, the TRACE-RA study [4] - a large placebo-controlled double-blind primary CV prevention trial in RA with atorvastatin was stopped prematurely owing to the very low number of CV events that occurred.

Altogether, the study from Desai and colleagues provides three important clues for improvement of
CV-RM in RA. First, more education is urgently needed for both rheumatologists and PCPs. Second, it is important to realise that the contribution of higher prevalence CRFs in RA is one side of the coin, but the other side is effective suppression of the inflammation. The latter is a clear task for the rheumatologist. Third, CV care of a RA patient should be a joint effort by the rheumatologist and the PCP, and they should collaborate and agree on who performs the screening, identification, and, if required, management of CRFs.

\section{Abbreviations}

CRF, cardiovascular risk factor; CV, cardiovascular; CV-RM, cardiovascular risk management; EULAR, European League Against Rheumatism; PCP, primary care physician; RA, rheumatoid arthritis.

\section{Competing interests}

The authors declare that they have no competing interests.

\section{Author details}

'Department of Internal Medicine, VU University Medical Centre, PO Box 7057, De Boelelaan 1117, 1081 HV Amsterdam, the Netherlands. ${ }^{2}$ Department of Rheumatology, VU University Medical Centre, PO Box 7057, De Boelelaan 1117 1081 HV Amsterdam, the Netherlands. ${ }^{3}$ Jan van Breemen Research Institute | Reade, Dr Jan van Breemenstraat 2, 1056 AB Amsterdam, the Netherlands.

\section{Published: 19 March 2013}

\section{References}

1. Desai SS, Myles JD, Kaplan MJ: Suboptimal cardiovascular risk factor identification and management in patients with rheumatoid arthritis: a cohort analysis. Arthritis Res Ther 2012, 14:R270.

2. Nurmohamed MT: Atherosclerotic disease in patients with inflammatory arthritis and systemic lupus erythematosus. In EULAR textbook on Rheumatic Diseases. Edited by Bijlsma JWJ, da Silva J, Hachulla E, Doherty M, Cope A Liotée F. London: BMJ Group; 2012:401-416.

3. Peters MJ, Symmons DP, McCarey D, Dijkmans BA, Nicola P, Kvien TK, McInnes IB, Haentzschel H, Gonzalez-Gay MA, Provan S, Semb A, Sidiropoulos P, Kitas G, Smulders YM, Soubrier M, Szekanecz Z, Sattar N, Nurmohamed MT: EULAR evidence-based recommendations for cardiovascular risk management in patients with rheumatoid arthritis and other forms of inflammatory arthritis. Ann Rheum Dis 2010, 69:325-331.

4. Trial of Atorvastatin for the Primary Prevention of Cardiovascular Events in Patients with Rheumatoid Arthritis

[http://www.dgoh.nhs.uk/tracera/Default.aspx/Home]

doi:10.1186/ar4185

Cite this article as: Peters MJL, Nurmohamed MT: Cardiovascular risk management in rheumatoid arthritis: are we still waiting for the first step? Arthritis Research \& Therapy 2013, 15:111. 